\title{
OBSERVATION AND CONTROL OF A SIMPLIFIED CAR
}

\author{
Guillaume, D. * Rouchon, P. ${ }^{* *}$ \\ * Centre Automatique et Systèmes, \\ École des Mines de Paris, \\ 60,boulevard Saint-Michel, \\ 75270 Paris Cedex, FRANCE \\ E-mail: guillaume@cas .ensmp.fr \\ ** idem. \\ E-mail: rouchon@cas .ensmp.fr
}

\begin{abstract}
The motion planning solution of a simplified car presented in (Fliess et al., 1995) admits a geometric formulation through the Frénet formulae. We present and use this formulation that preserves the invariance with respect to $S E(2)$ to built a global asymptotic observer of the non measured direction of the car via the only measurement of its position. Then we study the tracking of a planned path with an observer-controller. Although, the observer-controller stability proof is only local, the attraction domain seems to be very large as shown by numeric simulations (Matlab scripts are available from the authors via email). Copyright (c) 1998 IFAC
\end{abstract}

Keywords: flatness, symmetry, nonlinear observer, time scaling.

\section{INTRODUCTION}

The control of nonholomic vehicles was much studied in the past. In (Campion et al., 1996), a classification of such vehicles is proposed. The stabilization and the motion planning problem was also much studied. Stabilizing laws are proposed in (Canudas de Wit and Sørdalen, 1992; Sørdalen and Egeland, 1995; Rouchon et al., 1993; Walsh et al., 1994). The motion planning is studied in (Laumond et al., 1994; Rouchon et al., 1993; Tilbury et al., 1992). In this paper, we will study the problem of the observation and control of a class of these vehicles around a planned path using results presented in (Fliess et al., 1995; Rouchon et al., 1993). We adopt here a geometric point of view in the sense that all the computations steeming from (Fliess et al., 1995; Rouchon t al., 1993) are presented in an invariant manner via the Frénet frame [motion planning, tracking, observer] in order to preserve the structure of the system with respect to the Euclidean group $S E(2)$. In sections $2,3,4$, we present with the Frénet formulae an invariant solution of the motion planning design and tracking around the planned path. We then consider the problem of tracking this path when the only measurement is the position of the car. In the section 5, we present a reduced Luenbergerlike observer of the direction of the car via the only measurements of the position of the car. In the section 6 , we connect the controller and the reduced observer and do some remarks on the convergence and realization of the observercontroller. Simulations of section 7 illustrate robustness and the convergence domain.

\section{MODEL OF THE CAR}

We use here a well known model of the car (see e.g. (Campion et al., 1996) ) given by equations: 


$$
\left\{\begin{array}{l}
\dot{x}=u \cos \theta \\
\dot{y}=u \sin \theta \\
\dot{\theta}=\frac{u}{l} \tan \varphi
\end{array}\right.
$$

where $(x, y, \theta) \in \mathbb{R}^{2} \times \mathbb{S}^{1}$ is the state, $(u, \tan \varphi)$ is the control and $l$ is a positive parameter (length). It was established in (Fliess et al., 1995) that $x$ and $y$ are flat outputs. In Frénet coordinates, the system is described by the equations:

$$
\left\{\begin{array}{l}
\dot{\vec{P}}=v \vec{\tau} \\
\dot{\vec{\tau}}=v \frac{\tan \varphi}{l} \vec{\nu}
\end{array}\right.
$$

$\dot{f}$ represents the derivation of the function $f$ with respect to the time, $P=(x, y)$ is the position of middle of the back axis of the car (point in the plan), $v$ is the velocity of the car (first control), $\varphi$ the steering angle (second control) and $l$ the distance between the front and back axis of the car. $\vec{\tau}=(\cos \theta, \sin \theta)$ is the tangent vector of length 1 to the curve followed by $P$, and $\vec{\nu}=(-\sin \theta, \cos \theta)$, the normal vector to the curve such that $(\vec{\tau}, \vec{\nu})$ admits a positive orientation. We use here this representation in order to preserve the invariance of the system by the group of transformations $S E(2)$.

Notice also that the dynamics is invariant by time scaling. So we propose the following one:

$$
\bar{v} d s_{c}=v d t .
$$

where $s_{c}(t)$ correspond to an arc length parameterization [see below]. In the sequel, all the derivations are done according to this arc length. The main advantage is that all the computations can be done in a geometric frame, independent of the time $t$. The system is now described by:

$$
\left\{\begin{array}{l}
\vec{P}^{\prime}=\bar{v} \vec{\tau} \\
\vec{\tau}^{\prime}=\bar{v} \frac{\tan \varphi}{l} \vec{\nu}
\end{array}\right.
$$

where $(.)^{\prime}$ represents here the operator $\frac{d(.)}{d s_{c}}=$ $\frac{1}{\dot{s}_{c}} \frac{d(.)}{d t}$.

\section{MOTION PLANNING OF THE CAR}

Our goal is to plan the motion from an initial position $\left(P_{0}, \overrightarrow{\tau_{0}}\right)$ to a final position $\left(P_{f}, \overrightarrow{\tau_{f}}\right)$.

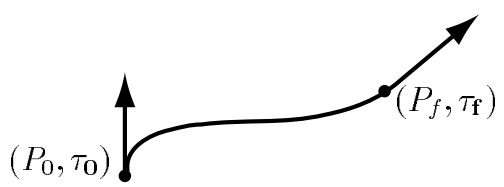

Figure 1. Planned path for the car.
We suppose we have a regular curve $P_{c}$, parameterized by the arc length $s_{c}$, such that $\left(P_{c}\left(s_{0}\right), \vec{\tau}_{c}\right.$ $\left.\left(s_{0}\right)\right)=\left(P_{0}, \vec{\tau}_{0}\right)$ and $\left(P_{c}\left(s_{f}\right), \vec{\tau}_{c}\left(s_{f}\right)\right)=\left(P_{f}, \vec{\tau}_{f}\right.$ ) . We then know all the geometric characteristics: $\vec{\tau}_{c}\left(s_{c}\right), \vec{\nu}_{c}\left(s_{c}\right), k_{c}\left(s_{c}\right)$ (curvature)...

We have the following planned motion:

$$
\left\{\begin{aligned}
\vec{P}_{c}^{\prime} & =\vec{\tau}_{c} \\
\vec{\tau}_{c}^{\prime} & =\frac{\tan \varphi_{c}}{l} \overrightarrow{\nu_{c}}=k_{c} \vec{\nu}_{c} \\
\bar{v}_{c} & =1 .
\end{aligned}\right.
$$

The reference arc length $s_{c}$ is chosen among arbitrary $C^{1}$ functions of the time such that:

$$
\left\{\begin{aligned}
{[0, T] } & \rightarrow\left[0, s_{f}\right] \\
t & \rightarrow s_{c}(t) \\
0 & \rightarrow 0 \\
T & \rightarrow s_{f}
\end{aligned}\right.
$$

If we add the condition on $\dot{s}_{c}$ so that the car starts at time $t=0$ from a steady state and stops at time $t=T$ at another steady state, we have: $\dot{s}_{c}(0)=\dot{s}_{c}(T)=0$.

Thus, we can easily obtain $v_{c}=\dot{s}_{c}(t)$ and $\tan \varphi_{c}=$ $l k_{c}$. The controls

$$
\left\{\begin{array}{l}
t \rightarrow v_{c}=\dot{s}_{c}(t) \\
t \rightarrow \varphi_{c(t)}=\arctan \left(l k_{c}\right) .
\end{array}\right.
$$

steer the car from the initial point $\left(P_{0}, \vec{\tau}_{0}\right)$ at $t=0$ to the final point $\left.\left(P_{f}, \overrightarrow{\tau_{f}}\right)\right)$ at $t=T$.

\section{CONTROLLER SYNTHESIS}

We use the flatness of the system to built the controller. From (Fliess et al., 1995), we know that $P$ is a flat output of the system. A linearizing feedback is obtained by standard input/output decoupling techniques via dynamic feedback. Presented with Frénet formulae, this leads to:

$$
\left\{\begin{aligned}
\vec{P}^{\prime \prime} & =\bar{w} \vec{\tau}+\bar{v}^{2} \frac{\tan \varphi}{l} \vec{\nu} \\
& =\vec{u} \\
\bar{v}^{\prime} & =\bar{w}
\end{aligned}\right.
$$

where $\vec{u}$ is the new control vector. We obtained a dynamic feedback with the introduction of the auxiliary state $\bar{v}$. With the control $\vec{u}$ set to:

$$
\vec{u}=\vec{P}_{c}^{\prime \prime}-\sigma_{1}\left(\vec{P}^{\prime}-\vec{P}_{c}^{\prime}\right)-\sigma_{2}\left(\vec{P}-\vec{P}_{c}\right)
$$

we obtain an error dynamics $\left(\stackrel{\vec{P}}{P}=\vec{P}-\vec{P}_{c}\right)$ :

$$
\tilde{\vec{P}}^{\prime \prime}+\sigma_{1} \tilde{\vec{P}}^{\prime}+\sigma_{2} \tilde{\vec{P}}=0
$$


$\sigma_{1}$ and $\sigma_{2}$ are parameters which are respectively homogeneous to $m^{-1}$ and $m^{-2}$ (inverse of a length and square of a length). We can then track the planned path and assign the dynamics through $\sigma_{1}$ and $\sigma_{2}$. The controls of the car are given by:

$$
\left\{\begin{aligned}
\bar{w} & =\vec{u} \cdot \vec{\tau} \\
\tan \varphi & =l \frac{\vec{u} \cdot \vec{\nu}}{\bar{v}^{2}}
\end{aligned}\right.
$$

We remark that locally around the planned path, $\bar{v}$ is in a neighborhood of 1 (because we have $\dot{s}_{c} \approx \dot{s}$ around the planned path). The above formulae are valid even at steady state i.e. when $v=0$, i.e. $\dot{s}_{c}=0\left(v=\bar{v} \dot{s}_{c}\right)$.

\section{OBSERVER SYNTHESIS}

The controller built in the section 4 , needs the measurements of ( $P, \vec{\tau})$ i.e. $(x, y, \theta)$. When only partial measurements of the state are available, it can not be used in the same way. In order to use the structure of the controller, we will estimate the non-measured variables thanks to an observer of $\vec{\tau}$ (or $\theta$ in the original coordinates). As in (Ribo et al., 1997), an extended Kalman filter can be used. We propose here another observer with global convergence and that remains invariant with respect to $S E(2)$.

We keep the same notations as in previous sections and built a purely geometric observer. This observer converge with a convergence rate, function of the covered length.

We consider a Luenberger-like reduced nonlinear observer with the introduction of the new auxiliary variable:

$$
\vec{\lambda}=\vec{\tau}+\frac{1}{a}\left(\vec{P}-\vec{P}_{c}\right)
$$

where $a$ is a constant different from 0 . We have:

$$
\vec{\lambda}^{\prime}=\bar{v} \frac{\tan \varphi}{l} \vec{\nu}+\frac{\bar{v}}{a}\left(\vec{\lambda}-\frac{1}{a}\left(\vec{P}-\vec{P}_{c}\right)-\vec{P}_{c}^{\prime}\right) .
$$

We built the following observer which is the copy of the dynamics of variable $\vec{\lambda}$ :

$$
\hat{\vec{\lambda}}^{\prime}=\bar{v} \frac{\tan \varphi \hat{\vec{\nu}}}{l}+\frac{\bar{v}}{a}\left(\hat{\vec{\lambda}}-\frac{1}{a}\left(\vec{P}-\vec{P}_{c}\right)-\vec{P}_{c}^{\prime}\right)
$$

where $\hat{\vec{\nu}}$ is the normal vector to $\hat{\vec{\tau}}=\hat{\vec{\lambda}}-\frac{1}{a}(\vec{P}$ $\left.-\vec{P}_{c}\right)$ such that $\|\hat{\vec{\tau}}\|=\|\hat{\vec{\nu}}\|$ and $(\hat{\vec{\tau}}, \hat{\vec{\nu}})$ admits a positive orientation. Due to input-output injection argument, the dynamics of the error $\tilde{\vec{\lambda}}=\vec{\lambda}-\hat{\vec{\lambda}}$ is given by:

$$
\tilde{\vec{\lambda}}^{\prime}=\bar{v} \frac{\tan \varphi}{l} \stackrel{\vec{\nu}}{l}+\frac{\bar{v}}{a} \overrightarrow{\vec{\lambda}} .
$$

We have $\tilde{\vec{\nu}} \cdot \tilde{\vec{\lambda}}=0(\tilde{\vec{\tau}}=\tilde{\vec{\lambda}})$. The derivative of $V=\|\tilde{\vec{\lambda}}\|^{2}$ along the trajectories is given by:

$$
V^{\prime}=\tilde{\vec{\lambda}}^{\prime} \cdot \tilde{\vec{\lambda}}=\frac{\bar{v}}{a}\|\tilde{\vec{\lambda}}\|^{2}
$$

The choice of the parameter $a$ allows us to make the derivative $V^{\prime}$ negative. This ensured the stability. Moreover, we have:

$$
\|V\|^{2} \leq\left\|V_{0}\right\|^{2} \exp \left(\int_{s_{0}}^{s} \frac{\bar{v}}{a} d s_{c}\right)
$$

So, as soon as $\int_{s_{0}}^{s} \frac{\bar{v}}{a} d s_{c} \rightarrow-\infty$ when $s_{c} \rightarrow+\infty$, we have asymptotic stability to zero.

\section{OBSERVER - CONTROLLER SYNTHESIS}

With the estimation of $\vec{\tau}$ and the measurements of $P$ and $\bar{v}$, we propose an observer-controller based on the controller (3) presented in the section 4 and on the observer (4) presented in the section 5 . We replace formally the non-measured variables by their estimates in the controller (3). We have thus:

$$
\left\{\begin{aligned}
\vec{u} & =\vec{P}_{c}^{\prime \prime}-\sigma_{1}\left(\hat{\vec{P}}^{\prime}-\vec{P}_{c}^{\prime}\right)-\sigma_{2}\left(\vec{P}-\vec{P}_{c}\right) \\
\overrightarrow{\vec{P}} & =\bar{v} \overrightarrow{\vec{\tau}}
\end{aligned}\right.
$$

The error dynamics is $(\tilde{\vec{\tau}}=\tilde{\vec{\lambda}})$ :

$$
\left\{\begin{aligned}
\tilde{\vec{\lambda}}^{\prime} & =\bar{v} \frac{\tan \varphi \tilde{\vec{\nu}}}{l}+\frac{\bar{v}}{a} \overrightarrow{\vec{\lambda}} \\
\tilde{\vec{P}}^{\prime \prime}+\sigma_{1} \tilde{\vec{P}}^{\prime}+\sigma_{2} \tilde{\vec{P}} & =\overline{\vec{v}}
\end{aligned}\right.
$$

Obviously, $(\tilde{\vec{P}}, \stackrel{\overrightarrow{\vec{P}}}{\prime}, \tilde{\vec{\lambda}})=(\overrightarrow{0}, \overrightarrow{0}, \overrightarrow{0})$ is an equilibrium point of the error dynamics. Locally around the planned path, we obtain by linearization a triangular structure, and the convergence proof is evident. Although the convergence is not ensured globally, simulations will show satisfactory results, even far away from the planned path.

Another problem is to avoid the "peacking" phenomena. It can appear when starting far from the actual values if $\bar{v}$ is equal to zero. This can be done thanks to the remark that locally around the planned trajectory $\bar{v}$ is in the neighborhood of 1 . We can change the control: 


$$
\left\{\begin{aligned}
\bar{w} & =\vec{u} \cdot \vec{\tau} \\
\tan \varphi & =l \frac{\vec{u} \cdot \vec{\nu}}{\max \left(\bar{v}^{2}, \gamma\right)}
\end{aligned}\right.
$$

where $\gamma$ is a positive number, small in comparison to 1 . Then $\tan \varphi$ and $\bar{v}$ are bounded and the state is also kept bounded.

\section{SIMULATIONS}

The following simulations illustrate the results of the previous sections. All the simulations are done with MATLAB and the m.files are available from the authors via email.

We assign a reference trajectory described in polar coordinates $\left(\rho_{c}, \alpha_{c}\right)$ by the parameterized equation $\rho_{c}=6+2 \cos \left(5 \alpha_{c} / 2\right)$. The trajectory in Euclidean coordinates is given by: $P_{c}=$ $\left(\rho_{c} \cos \left(\alpha_{c}\right), \rho_{c} \sin \left(\alpha_{c}\right)\right)$. We chose: $\alpha_{c}(t)=\sigma\left(\frac{t}{T}\right) 4 \pi$ with $\sigma$ given by: $\sigma(\mu)=3 \mu^{2}-2 \mu^{3}$. We have: $\dot{s}_{c}=\sqrt{\rho^{2}+\rho^{2}} \dot{\alpha}_{c}$. The length $l$ between the two axis of the car is $l=2 \mathrm{~m}$. The simulation time $T$ is set to $T=1$ so that the car can achieve one period of the cycle. The initial condition for $\bar{v}$ will be set to 1 according to the fact that the actual value is in the neighborhood of 1 very quickly.

The open loop control of the car is easily computed thanks to the flatness of the system. The closed trajectory is displayed on figure 2 .

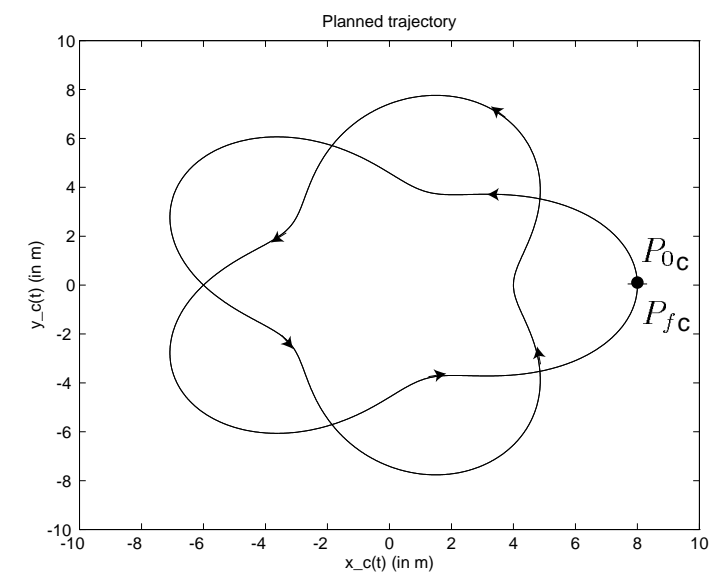

Figure 2. Open loop control of the car.

Let us look closer at the performance of the observer-controller. We set the gains of the controller $\sigma_{1}$ and $\sigma_{2}$ to $\left(\sigma_{1}, \sigma_{2}\right)=(2 / 5,1 / 25)$. The observer gain $a$ is set to $a=-1$, so that the convergence of the observer is quicker than the one of the controller. The initial conditions of the system, observer and reference trajectory are given by:

$$
\left\{\begin{aligned}
x_{0} & =8, \quad y_{0}=8, \quad \theta_{0}=\pi / 2 \\
\hat{x}_{0} & =8, \quad \hat{y}_{0}=8, \quad \hat{\theta}_{0}=3 \pi / 4 \\
x_{0 c} & =8, y_{0 c}=0, \theta_{0 c}=\pi / 2
\end{aligned}\right.
$$

The results of the convergence are illustrated on the figure 3 . Then we add an error of $20 \%$

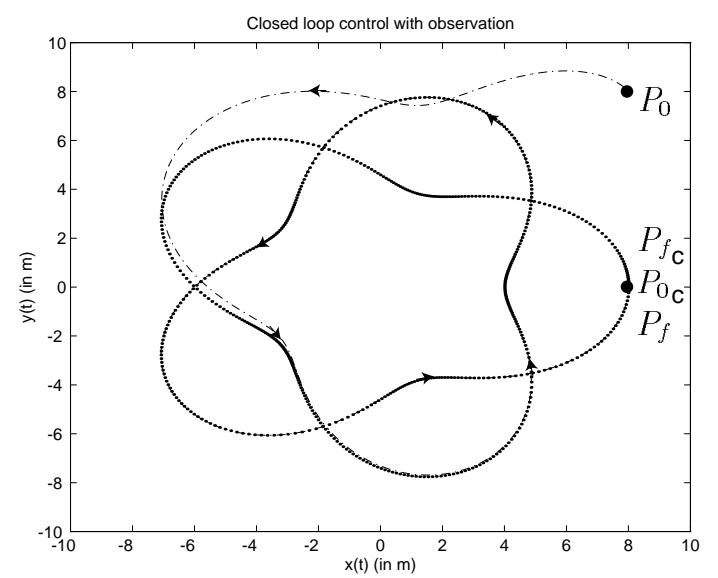

Figure 3. Control of the car by the observercontroller with an error on the initial conditions (dotted: reference, dash-dotted: car trajectory).

on the parameter $l$ and keep the same initial conditions (5). We see on the figure 4 that the performance are still good and that the tracking error is maximum when curvature is maximum.

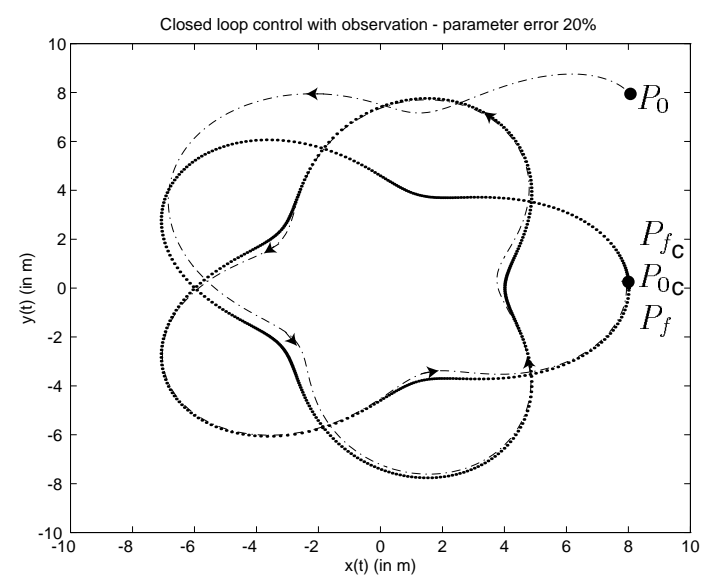

Figure 4. idem as on the figure 3 with parameter error (dotted: reference, dash-dotted: car trajectory).

\section{CONCLUSION}

The observer-controller presented here is particularly simple. Most of this simplicity is due to an intensive use of Frénet formulae leading to simple equations and invariant computations. Symmetries have already been used for the design of controllers in the past (see e.g. (Grizzle and Marcus, 1985; Hazewinkel and Martin, 1983; van der 
Schaft, n.d.; Fagnani and Willems, 1993)). This paper shows that symmetry can also be used for observer design: observer (4) is clearly invariant.

\section{REFERENCES}

Campion, G., B. d'Andréa Novel and G. Bastin (1996). Structural properties and classification of kinematic and dynamic models of wheeled mobile robots. IEEE Trans. robotics Automation 12, 47-62.

Canudas de Wit, C. and O.J. Sørdalen (1992). Exponential stabilization of mobile robots with nonholonomic constraints. IEEE Trans. Automat. control 37, 1791-1797.

Fagnani, F. and J. Willems (1993). Representations of symmetric linear dynamical systems. SIAM J. Control and Optim. 31, 1267-1293.

Fliess, M., J. Lévine, P. Martin and P. Rouchon (1995). Flatness and defect of nonlinear systems: introductory theory and examples. Int. J. Control 61, 1327-1361.

Grizzle, J.W. and S.I. Marcus (1985). The structure of nonlinear control systems possessing symmetries. IEEE Trans. Automat, control 30, 248-258.

Hazewinkel, M. and C.F. Martin (1983). Symmetric linear systems: An application of algebraic systems theory. Int. J. Control 37, 13711384 .

Laumond, J.P., Jacobs P., M. Tax and M. Murray (1994). A motion planner for nonholonomic mobile robots. IEEE Transactions on robotics and Automation 10, 577-593.

Ribo, M., S. Brahim-Belhouari and L. Pronzato (1997). Motion planning for the precise location of a mobile robot. Proc. ECC'97, Bruxelles.

Rouchon, P., M. Fliess, J. Lévine and P. Martin (1993). Flatness and motion planning: the car with n trailers. Proc. ECC'93, Groningen pp. 1518-1522.

Sørdalen, O.J. and O. Egeland (1995). Exponential stabilization of nonholonomic chained systems. IEEE Trans. Automat. Control 40, 35-49.

Tilbury, D., J.P. Laumond, R. Murray, S. Sastry and G. Walsh (1992). Steering car-like systems with trailers using sinusoids. Proceedings IEEE International conf. on robotics and Automation pp. 1993-1198.

van der Schaft, A.J. (n.d.). Symmetries in optimal control. SIAM J. Control Optim. 25, 245259 .

Walsh, G., D. Tilbury, S. Sastry, R. Murray and J.P. Laumond (1994). Stabilization of trajectories for systems with nonholonomic constraints. IEEE trans. Automat. Control $39,216-222$. 